\title{
A Low-Rate Identification Method for Digital Predistorters Based on Volterra Kernel Interpolation
}

\author{
Peter Singerl ${ }^{1,2}$ and Heinz Koeppl ${ }^{1}$ \\ ${ }^{1}$ Christian Doppler Laboratory for Nonlinear Signal Processing \\ Graz University of Technology, Austria \\ ${ }^{2}$ Infineon Technologies Austria AG \\ Email: \{peter.singerl, heinz.koeppl\}@tugraz.at
}

\begin{abstract}
RF power amplifiers (PA) of wireless communication systems are usually driven in their nonlinear region to obtain a higher efficiency. The price paid for the increased efficiency is the distortion of the transmission signal. To fulfill a given spectral mask for the transmission signal and to avoid an unacceptable high bit-error rate on the receiver side, the amplifier must be linearized. It is well known that nonlinear system identification (PA or predistorter) can be accomplished on the Nyquist-rate regarding the input signal bandwidth. However for predistortion applications, systems operating on the output signal Nyquist-rate are required. In this paper we show how such predistortion systems can be obtained by a multi-dimensional Volterra kernel interpolation where the kernels are estimated on a Nyquist-rate regarding the input signal, without the demand for an expensive high sampling rate analog to digital converter (ADC).
\end{abstract}

\section{INTRODUCTION}

To operate power transmitters of RF communication systems with a higher efficiency, the final PA output-stages are usually driven in their nonlinear region (approximate inverse relationship between amplifier efficiency and linearity). The price paid for a higher efficiency is that the nonlinearity of the PA causes in-band distortion, which degrades the bit-error performance. It also causes spectral regrowth which leads to neighbor channel interference. Newer modulation formats such as WCDMA or OFDM are especially vulnerable to nonlinearities due to their high peak to average power ratio. In order to comply with spectral masks imposed by regulatory bodies and to reduce the bit error rate, the PA is linearized. The most powerful and efficient linearization technique is digital baseband predistortion [1] [4]. Figure 1 depicts a simplified block diagram of a communication transmitter. The digital baseband predistorter distorts the input signal by a nonlinear operator which is ideally the inverse nonlinearity of the PA to obtain an almost overall linear response up to the saturation point. To compensate the spectral out-off-band components caused by the amplifier nonlinearity, the predistorter has to be operated on a sufficient high sampling frequency $\omega_{s}$ which is usually $2 P$-times higher than the transmission signal bandwidth. The factor $P$ denotes the highest odd-order nonlinearity of the PA. Because the nonlinear behavior of the PA is in general unknown, we apply a feedback path in Fig. 1 in order to identify either the amplifier and compute the inverse (predistorter) or to identify immediately the predistorter [5]-[8]. It is well known that the identification process can be accomplished on a sampling frequency which is just twice the input signal bandwidth [9]. Based on this result, we apply a Volterra kernel interpolation on the low-rate identified kernels. Therefore we can reduce the sampling rate of the feedback path $\omega_{F}=\omega_{S} / P$, compared to the traditional approach.

II. COMPLEX BASEBAND MODELING OF NONLINEAR PASSBAND SYSTEMS

Nonlinear passband systems like RF PAs can be modeled by a tandem connection of a dynamic nonlinear system (real Volterra

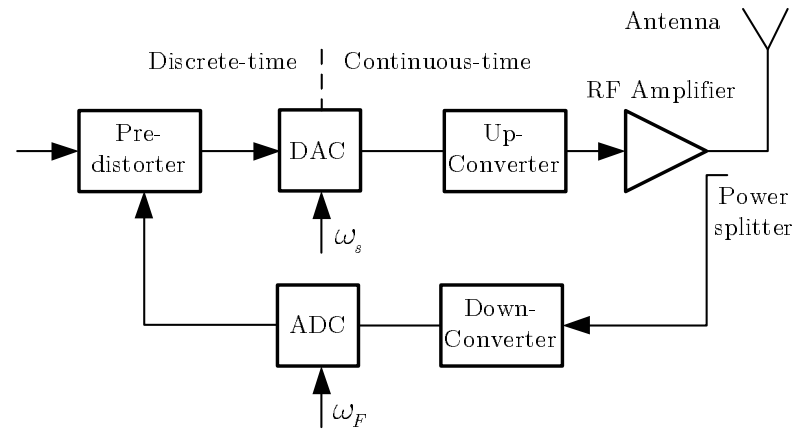

Fig. 1. Block diagram of a wireless communication transmitter with digital baseband predistortion for the linearization of the RF amplifier.

series) and a linear passband filter (1st-zonal filter) to suppress the unwanted spectral components located around the multiples of the RF carrier frequency $\omega_{c}$ [10]. If $\omega_{c}>B(2 P-1)$, where $B$ describes the input signal bandwidth, an equivalent baseband Volterra system exists, which generates the filtered and frequency shifted output signal of the real PA directly in the complex baseband domain. Such baseband systems are preferable used, e.g., to derive a sufficiently accurate predistortion structure or to predict the performance of such a system without the full computational complexity of a transistorlevel based simulator. Both the PA model and the predistorter has to be operated on a sufficient high sampling frequency $\omega_{s}$ which is at least $2 P$-times higher (Nyquist theorem regarding the PA output signal bandwidth) than the transmission signal bandwidth to generate the corresponding spectral out-off-band components of the real PA without any aliasing effects. The complex discrete-time PA Volterra model can be described in operator notation by

$$
y(n)=\mathbf{H}_{1}[x(n)]+\underbrace{\sum_{p=1}^{\lceil P / 2\rceil-1} \mathbf{H}_{2 p+1}[x(n)]}_{\mathbf{H}_{(P)}},
$$

with the functional description

$$
\begin{array}{r}
\mathbf{H}_{2 p+1}[x(n)]=\sum_{k_{1}=0}^{M-1} \ldots \sum_{k_{2 p+1}=0}^{M-1} h_{2 p+1}\left(k_{1}, \ldots, k_{2 p+1}\right) \\
\cdot \prod_{i=1}^{p+1} x\left(n-k_{i}\right) \prod_{i=p+2}^{2 p+1} x^{*}\left(n-k_{i}\right),
\end{array}
$$

where $x, y$ and $h_{2 p+1}$ describes the complex baseband input and output signals and the baseband equivalent time-domain Volterra kernels respectively. $P \in \mathbb{N}_{o}$ describes the highest order of the baseband PA nonlinearity and "*" denotes complex conjugation. 
It is important to note that due to the bandpass-nature of the real PA input signal $x(t)=A(t) \cos \left(\omega_{c} t+\phi_{0}(t)\right)$, where $A(t)$ denotes the $\mathrm{AM}$ and $\phi_{0}(t)$ denotes the PM, only odd-order terms appears in the baseband model in (1) [10]. The complex baseband Volterra kernels $h_{2 p+1}$ in (2) are related to the odd-order real Volterra kernels by a magnitude scaling and a phase shift [10].

\section{PRedistortion STRUCTURE}

A predistorter is a nonlinear operator which precedes the PA in order to distort the incoming PA signal to obtain ideally an overall linear system response of the cascade of predistorter and PA. In [11] it is shown that for a certain class of nonlinear systems an exact inverse exists. However, for most of the nonlinear systems we are not able to find an exact inverse [12]. Many researchers have found different approximate system inverses with memory which are based either on PA modeling and predistorter calculation or direct predistorter estimation [5]-[8], [12]-[14].

In this paper we focus on a simple predistortion structure proposed in [8] which is composed of a parallel structure of a delay and a cascade of a nonlinear and a linear filter. To estimate the linearization performance of this predistortion architecture which is also depicted in Fig. 5, the overall system denoted by the operator

$$
\mathbf{L}=\underbrace{\left(\mathbf{H}_{1}+\mathbf{H}_{(P)}\right)}_{\text {PA }} \circ \underbrace{\left(\mathbf{D}+\breve{\mathbf{H}}_{1}^{-1} \circ \breve{\mathbf{H}}_{(P)}\right)}_{\text {Predistorter }}
$$

has to be described in terms of its set of frequency-domain Volterra kernels $\left\{L_{2 p+1}\left(z_{1}, \ldots, z_{2 p+1}\right)\right\}$ for $p=0,1,2, \ldots,\left\lceil P^{2} / 2-1\right\rceil$. The operator $\mathbf{D}$ delays the complex input signal $x$ at $\delta$ samples to compensate the latency of the cascade of $H_{1} \circ \breve{H}_{1}^{-1}$ where the symbol "०" describes the cascade of two Volterra systems $(\mathbf{A} \circ \mathbf{B})[x]=\mathbf{A}[\mathbf{B}[x]]$. If we apply the frequency-domain Volterra kernel calculation rule for a cascade (predistorter and PA model) of two discrete-time systems [15] and arrange the indices in order to consider only the present odd-order Volterra kernels we obtain

$$
\begin{aligned}
& L_{2 p+1}\left(z_{1}, \ldots, z_{2 p+1}\right)=\sum_{n=0}^{p} \underbrace{\sum_{m_{1}=1} \ldots \sum_{m_{2 n+1}=1}}_{\substack{u(2 n+1)=2 p+1 \\
m_{i} \in \mathbb{N}_{o}}}\{ \\
& H_{2 n+1}\left(z_{l(1)} \cdots z_{u(1)}, \ldots, z_{l(2 n+1)} \cdots z_{u(2 n+1)}\right) \\
& \left.\cdot \prod_{k=1}^{2 n+1} \breve{H}_{1}^{-1}\left(z_{l(k)} \cdots z_{u(k)}\right) \breve{H}_{m_{k}}\left(z_{l(k)}, \ldots, z_{u(k)}\right)\right\} \text {, }
\end{aligned}
$$

where the lower and upper index bound functions are defined by $l(k)=l\left(m_{1}, \ldots, m_{k-1}\right)=1+\sum_{j=1}^{k-1} m_{j}$, and $u(k)=$ $u\left(m_{1}, \ldots, m_{k}\right)=\sum_{j=1}^{k} m_{j}$, respectively with $l(1)=1$. If we separate the terms in (4) for $n=0$ and $n=p$ we obtain with $\breve{H}_{1}(z) \breve{H}_{1}^{-1}(z)=z^{-\delta}$

$$
\begin{aligned}
& L_{2 p+1}\left(z_{1}, \ldots, z_{2 p+1}\right)=H_{1}\left(z_{1} \cdots z_{2 p+1}\right) \breve{H}_{1}^{-1}\left(z_{1} \cdots z_{2 p+1}\right) \\
& \cdot \breve{H}_{2 p+1}\left(z_{1}, \ldots, z_{2 p+1}\right)+H_{2 p+1}\left(z_{1}, \ldots, z_{2 p+1}\right) \\
& \cdot\left(z_{1} \cdots z_{2 p+1}\right)^{-\delta}+\sum_{n=1}^{\sum^{-1} \underbrace{\sum_{1}=1}_{\substack{u(2 n+1)=2 p+1 \\
m_{i} \in \mathbb{N}_{o}}} \sum_{m_{2 n+1}=1}}\{ \\
& H_{2 n+1}\left(z_{l(1)} \cdots z_{u(1)}, \ldots, z_{l(2 n+1)} \cdots z_{u(2 n+1)}\right) \\
& \left.\cdot \prod_{k=1}^{2 n+1} \breve{H}_{1}^{-1}\left(z_{l(k)} \cdots z_{u(k)}\right) \breve{H}_{m_{k}}\left(z_{l(k)}, \ldots, z_{u(k)}\right)\right\},
\end{aligned}
$$

where the first two summands in (5) (cross pathes $\mathbf{H}_{(P)} \circ \mathbf{D}$ and $\mathbf{H}_{1} \circ \breve{\mathbf{H}}_{1}^{-1} \circ \breve{\mathbf{H}}_{(P)}$ in Fig. 5) equalize if the linear frequency-domain kernel of the predistorter

$$
\breve{H}_{1}(z)=-H_{1}(z) z^{\delta}
$$

and the $2 p+1$-dimensional frequency-domain kernels for $p \geq 1$ are

$$
\breve{H}_{2 p+1}\left(z_{1}, \ldots, z_{p}\right)=H_{2 p+1}\left(z_{1}, \ldots, z_{p}\right) .
$$

Thus the system with a perfectly adjusted predistorter results in the following odd-order frequency-domain Volterra kernels

$$
\begin{aligned}
& L_{1}(z)=H_{1}(z) z^{-\delta} \\
& L_{3}\left(z_{1}, z_{2}, z_{3}\right)=0 \\
& L_{2 p+1}\left(z_{1}, \ldots, z_{2 p+1}\right)=-\sum_{n=1}^{p-1} \underbrace{\sum_{m_{1}=1} \ldots \sum_{m_{2 n+1}=1}}_{\begin{array}{c}
u(2 n+1)=2 p+1 \\
m_{i} \in \mathbb{N}_{o}
\end{array}}\{ \\
& \cdot H_{2 n+1}\left(z_{l(1)} \cdots z_{u(1)}, \ldots, z_{l(2 n+1)} \cdots z_{u(2 n+1)}\right) \\
& 2 n+1 \\
& \cdot \prod_{k=1}^{2 n+1} H_{1}^{-1}\left(z_{l(k)} \cdots z_{u(k)}\right)\left(z_{l(k)} \cdots z_{u(k)}\right)^{-\delta} \\
& \left.\breve{H}_{m_{k}}\left(z_{l(k)}, \ldots, z_{u(k)}\right)\right\} \text {. }
\end{aligned}
$$

Although perfect equalization is only possible for the third order Volterra kernel $L_{3}\left(z_{1}, z_{2}, z_{3}\right)$, the resulting distortion of the predistorted PA output signal is much lower compared to the noncompensated PA if $\left|\mathbf{H}_{1}[x(n)]\right| \gg\left|\mathbf{H}_{(P)}[x(n)]\right|$ because all the kernels $L_{2 p+1}$ for $p \geq 2$ in (8) incorporates the multiplicative inverse of the linear PA kernel $H_{1}^{-1}$.

\section{Predistorter identificAtion AND VolterRa KeRnel INTERPOLATION}

The predistortion architecture we have investigated in the last section has to work on a sufficiently high sampling rate (Nyquist theorem regarding the PA output signal) to be able to compensate the spectral out-off-band components generated by the PA. It is shown in [9] that although the PA output signal occupies the normalized frequency range from $\pm \pi$, the PA can be identified from its frequencydomain kernels over the normalized input signal bandwidth of $\pi / P$. To investigate this in detail we transform the time-domain signals from (2), with $n \rightarrow n_{1}, n_{2}, \cdots, n_{2 p+1}$ to the frequency-domain by applying a multi-dimensional $\mathcal{Z}$-transform, which yields

$$
\begin{aligned}
& Y_{2 p+1}\left(z_{1}, \ldots, z_{2 p+1}\right)=H_{2 p+1}\left(z_{1}, \ldots, z_{2 p+1}\right) \\
& \cdot \prod_{i=1}^{p+1} X\left(z_{i}\right) \prod_{i=p+2}^{2 p+1} X^{*}\left(z_{i}^{*}\right) .
\end{aligned}
$$

The multi-dimensional output signals $Y_{2 p+1}$ are band-limited if the input signal $X$ is band-limited. Therefore the PA is uniquely defined by the Volterra kernels $H_{2 p+1}$ within the input signal bandwidth, because the kernels outside are masked by the band-limited input signals $X$. This process is depicted in Fig. 2 for a two-dimensional kernel for $P=2$, which was chosen for a convenient graphical representation although we do not have even-order kernels in passband systems considered here. The shaded areas in Fig. 2 represents the non-zero values of the frequency-domain kernels. The identification process of the PA described by the operators $\mathbf{H}_{1}$ and $\mathbf{H}_{(P)}$ in Fig. 5 is accomplished with an adaptive algorithm on the low-rate with the task 
to minimize a certain cost function. Therefore we only need a lowrate $\mathrm{ADC}$ in the analog front-end for the identification process, which save costs compared to the traditional high-rate approach. Because the nonlinear operator $\mathbf{H}_{(P)}$ of the PA is operated in the predistorter on the band-limited input signal of $\pi / P$, we can easily recalculate the low-rate identified nonlinear operator $\hat{\mathbf{H}}_{(P)}$ to a high rate model $\breve{\mathbf{H}}_{(P)}$ by a multi-dimensional zero padding (interpolation) [16]. The unwanted spectral copies caused by the zero-padding are masked by the band-limited high-rate input signal $X$. This operation can be accomplished with almost no additional costs (each unit-sample delay is replaced by a $P$-sample delay). The zero padding in the time domain results in a simple frequency scaling in each of the $2 p+1$ dimension which yields

$$
\breve{H}_{2 p+1}\left(z_{1}, \ldots, z_{2 p+1}\right)=\hat{H}_{2 p+1}\left(z_{1}^{P}, \ldots, z_{2 p+1}^{P}\right) .
$$

The frequency-domain output signal of the estimated nonlinear operator $\breve{\mathbf{H}}_{(P)}$ is given with (9) and (10) by the contour integral [15]

$$
\begin{aligned}
& \breve{Y}(z)=\sum_{p=0}^{\lceil P / 2\rceil-1} \frac{1}{(j 2 \pi)^{2 p}} \\
& \oint_{C} \cdots \oint_{C} \frac{\breve{Y}_{2 p+1}\left(z_{1}, z_{2} / z_{1}, \ldots, z / z_{2 p}\right)}{z_{1} \ldots z_{2 p}} d z_{1} \ldots d z_{2 p},
\end{aligned}
$$

which spreads the spectrum from $\pi / P$ to $\pi$ if (11) is evaluated on the unit-circle in the complex plane. If the linear kernel $H_{1}\left(e^{j \omega}\right)$ of the PA is not absolutely flat, than $\breve{H}_{1}^{-1}\left(e^{j \omega}\right) \neq H_{1}^{-1}\left(e^{j \omega}\right)$ for $|\omega|>$ $\pi / P$. This can cause an imperfect equalization for the out-off-band spectrum $(\omega>\pi / P)$, because the spectrum is complex weighted with the periodically $(2 \pi / P)$ continued linear kernel $\breve{H}_{1}^{-1}\left(e^{j \omega}\right)$, which differs from the desired kernel $H_{1}^{-1}\left(e^{j \omega}\right)$. If a stronger neighbor channel power suppression is required than we can achieve with a purely interpolation of the linear kernel, we can additionally adjust this kernel with a low-rate adaptive algorithm (c.f. Fig. 5).

\section{Simulation Results}

We have simulated the predistortion structure depicted in Fig. 5 with a 5th-order complex discrete-time PA model shown in Fig. 3. The higher order Volterra kernels of this reference model obey a special structure and are given in their symmetrized versions as $H_{3}\left(z_{1}, z_{2}, z_{3}\right)=\frac{1}{3 !} \sum_{\pi \in \mathfrak{S}^{3}} \tilde{H}_{3}\left(z_{\pi_{1}}, z_{\pi_{2}}, z_{\pi_{3}}\right)$ with $\tilde{H}_{3}\left(z_{1}, z_{2}, z_{3}\right)=H_{34}\left(z_{1} z_{2} z_{3}\right) H_{31}\left(z_{1}\right) H_{32}\left(z_{2}\right) H_{33}\left(z_{3}\right)$ where $\mathfrak{S}^{3}$ denotes the set of all permutations of a 3 -tuple and $H_{5}\left(z_{1}, \ldots, z_{5}\right)=$ $a$. This nonlinear system is depicted within the dashed-line box in Fig. 3. If the non-predistorted PA is excited with a band-limited input signal $(B \approx \pi / 5)$, we obtain a spectral regrowth by $P=5$, which is depicted in Fig. 4. If we apply a predistorter which precedes the PA, we are able to reduce the nonlinear in- and out-off-band distortion. The power suppression outside the signal bandwidth depends on the flatness of the linear kernel $H_{1}$ of the PA. In this simulation we applied a linear kernel with magnitude variations around $1.5 \mathrm{~dB}$. In this case we can reduce the out-off-band power in the neighbor channel by approximately $10 \mathrm{~dB}$ (linearized PA output signal with $\hat{H}_{1}^{-1}$ in Fig. 4). This distortion can be further reduced $(10 \mathrm{~dB})$ if we apply an additional low-rate adaptive algorithm to adjust the interpolated linear kernel $\breve{H}_{1}$ over the whole frequency band of $\pm \pi$ (linearized PA output signal with $\breve{H}_{1}^{-1}$ in Fig. 4).

\section{CONCLUSION}

Although nonlinear systems can be identified on a sampling rate which is just twice the input signal bandwidth, for digital predistortion

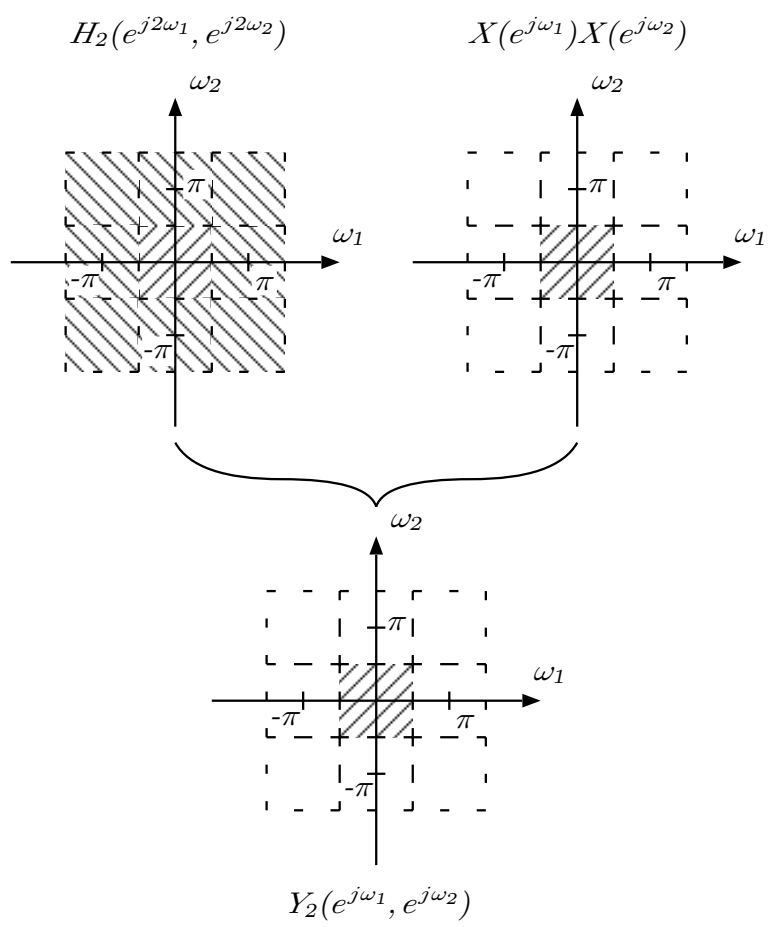

Fig. 2. Two-dimensional zero-padding and spectral masking by the highrate input-signal $X\left(e^{j \omega_{1}}\right) X\left(e^{j \omega_{2}}\right)$ for a two-dimensional Volterra kernel The shaded areas illustrates the non-zero frequency-domain kernels.

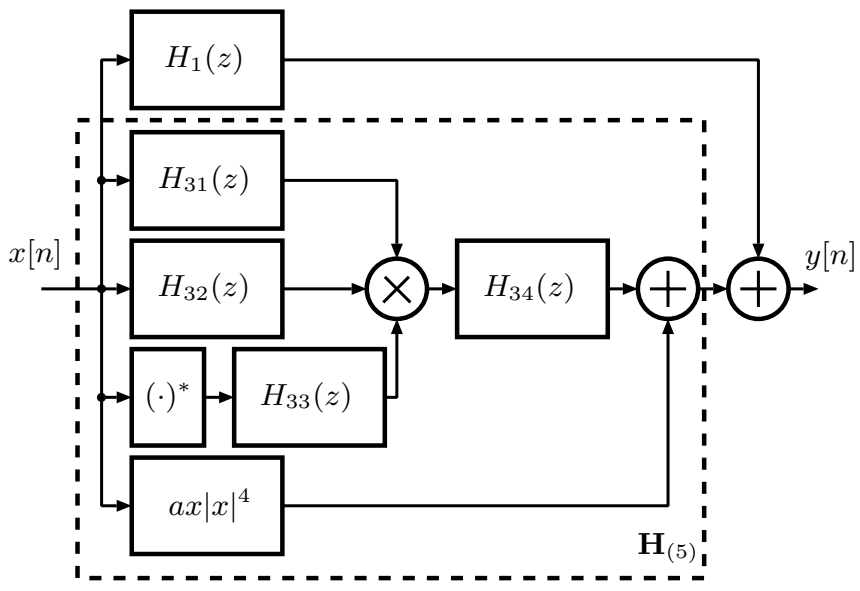

Fig. 3. 5th-order nonlinear PA model which is composed of a linear filter and a 5th-order nonlinear Volterra filter which is inside the dashed-line box.

a sampling rate which is at least twice the output signal bandwidth is required. However we have shown for a particular predistortion architecture that is sufficient to interpolate the nonlinear kernels of the low-rate identified PA and copy these high-rate kernels into the predistorter. The unwanted spectral copies of the higher order kernels are masked out by the band-limited input signals. This multi-rate predistorter works perfect (compared to the purely high-rate system) if the linear kernel of the PA is flat (interpolated linear kernel is equally the high-rate identified kernel), otherwise we have a suboptimal solution which converges to the ideal one, if we apply an additional low-rate adaptive algorithm to adjust the frequency response of the interpolated linear kernel in the predistorter over 


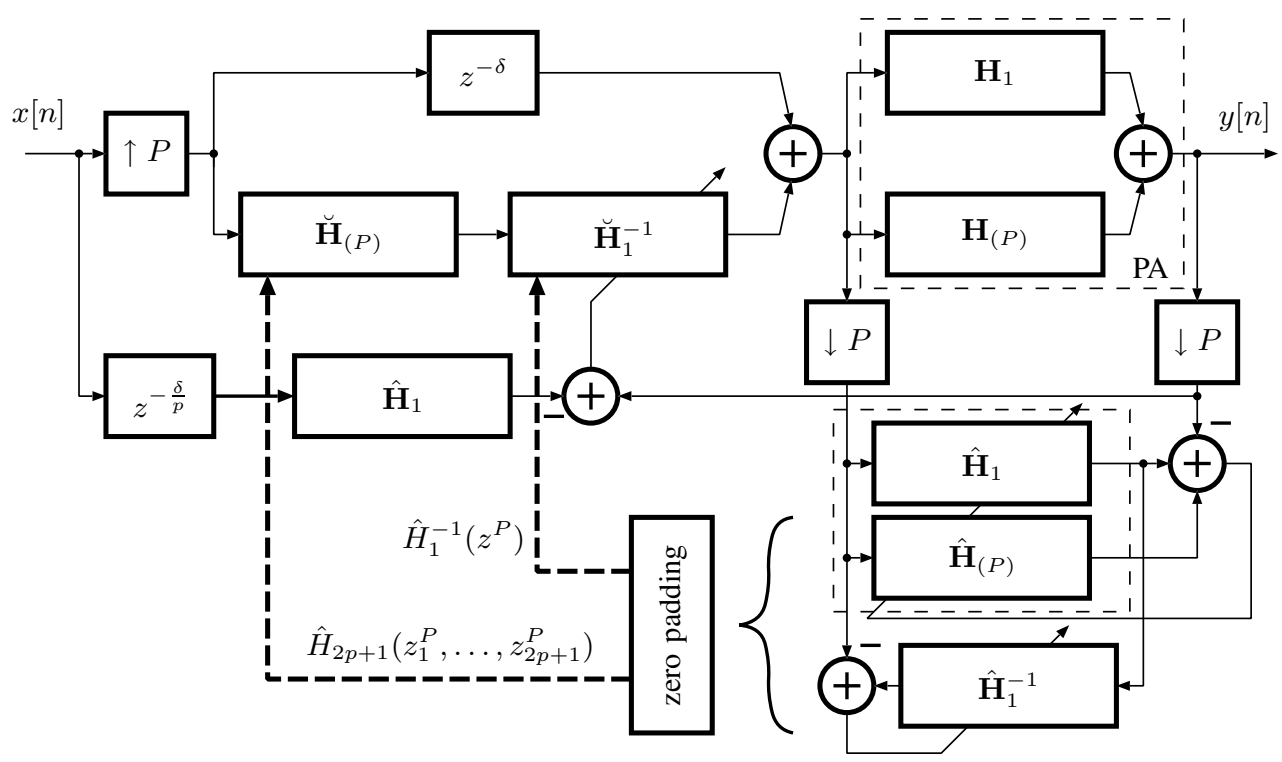

Fig. 5. Multi-rate digital predistortion architecture composed of a high-rate PA model $\mathbf{H}_{1}+\mathbf{H}_{(P)}$, high-rate predistorter $\mathbf{D}+\breve{\mathbf{H}}_{1}^{-1} \otimes \breve{\mathbf{H}}_{(P)}$, low-rate system identification and Volterra kernel interpolation (zero padding).

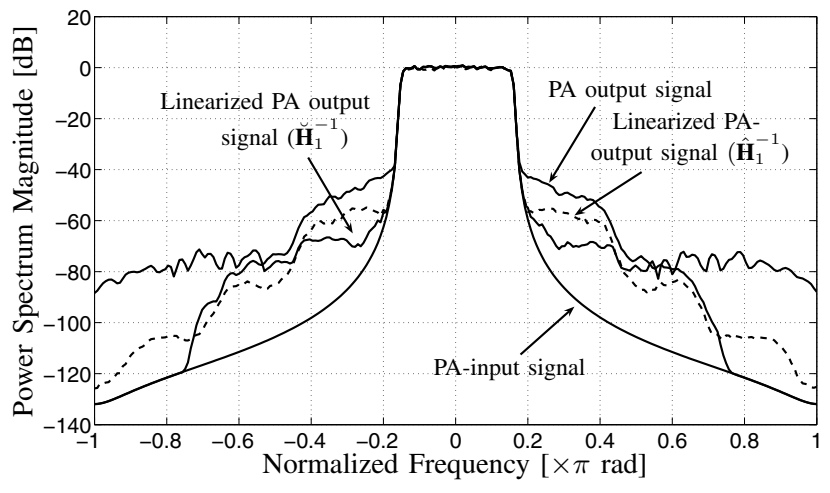

Fig. 4. Input- and output-spectra of the non-linearized and linearized power amplifier. The linearization is accomplished with the two different linear systems $\hat{\mathbf{H}}_{1}^{-1}$ (interpolated low-rate kernel) and $\breve{\mathbf{H}}_{1}^{-1}$ (low-rate adaptive identified kernel) in Fig. 5.

the whole frequency-band. With a typical smooth linear kernel for the PA (magnitude variation is approximately $1.5 \mathrm{~dB}$ ) we obtain a neighbor channel power suppression of approximately $10 \mathrm{~dB}$ if we solely apply Volterra kernel interpolation on the low-rate identified kernels, and $20 \mathrm{~dB}$ if we additionally adjust the frequency response of the interpolated linear kernel over the whole frequency band. This performance can be obtained, although we only employ a low-cost, low-rate $\mathrm{ADC}$ in the analog front-end of the transmitter feedback path.

\section{ACKNOWLEDGMENT}

The authors would like to thank D. Schwingshackl and C. Vogel from the Technical University Graz for their helpful comments.

\section{REFERENCES}

[1] Peter B. Kennington, High linearity RF amplifier design, Artech House, Boston, London, 2000.
[2] Steve C. Cripps, RF power amplifiers for wireless communications, Artech House, Boston, London, 1999.

[3] Steve C. Cripps, Advanced techniques in RF power amplifier design, Artech House, Boston, London, 2002.

[4] James K. Cavers, "Amplifier linearization using a digital predistorter with fast adaption and low memory requirenments," IEEE Trans. Veh. Technol., vol. 37, pp. 1885-1890, Dec. 1989.

[5] Raviv Raich, Hua Qian, and G. T. Zhou, "Digital baseband predistortion of nonlinear power amplifiers using orthogonal polynomials," in IEEE International Conference on Acoustics, Speech, and Signal Processing, 2003. Proceedings. (ICASSP '03), Apr. 2003, pp. 689-692.

[6] Lei Ding, Raviv Raich, and G. T. Zhou, "A Hammerstein predistortion linearization design based on the indirect learning architecture," in IEEE International Conference on Acoustics, Speech, and Signal Processing, 2002. Proceedings. (ICASSP '02), May 2002, pp. 2689-2692.

[7] Qian Yeqing, Li Qi, and Yao Tianren, "Analysis of different predistortion structures and efficient least-square adaptive algorithms," IEEE International Conference on Acoustics, Speech and Signal Processing, Apr. 2003, vol. 2, pp. 461-464.

[8] Xiang Yang Gao and W. Martin Snelgrove, "Adaptive linearization schemes for weakly nonlinear systems using adaptive linear and nonlinear FIR filters," in Midwest Symposium on Circuits and Systems, Aug. 1990, pp. 9-12.

[9] Walter A. Frank, "Sampling requirements for Volterra system identification," IEEE Signal Processing Letters, vol. 3, pp. 266-268, Sept. 1996.

[10] S. Benedetto, E. Biglieri, and R. Daffara, "Modeling and performance evaluation of nonlinear satellite links—a Volterra series approach," IEEE Trans. Aerosp. Electron. Syst., vol. 15, pp. 494-507, July 1979.

[11] V. John Mathews and Giovanni L. Sicuranza, Polynomial signal processing, John Wiley \& Sons, INC, 2000.

[12] Martin Schetzen, The Volterra and Wiener theories of nonlinear systems, Krieger publishing company, Malabar, Florida, 1980.

[13] J. Kim and K. Konstantinou, "Digital predistortion of wideband signals based on power amplifiers with memory," Electronics Letters, vol. 37 , pp. 1417-1418, Nov. 2001.

[14] A. Zhu, M. Wren, and T.J. Brazil, "An efficient Volterra-based behavioral model for wideband RF power amplifiers," in Microwave Symposium Digest, IEEE MTT-S international, June 2003, pp. 787-790.

[15] Wilson J. Rugh, Nonlinear system theory, The Johns Hopkins University Press, 2002.

[16] Peter Singerl and Heinz Koeppl, "Volterra kernel interpolation for system modeling and predistortion purposes," in IEEE International Symposium on Signals Circuits and Systems, 2005. Proceedings. (ISSCS '05), July 2005, to be published. 